\section{Avaliação da satisfação dos usuários com a qualidade do atendimento nas grandes emergências do Recife, Pernambuco, Brasil}

\author{
Assessing user satisfaction regarding the \\ quality of health care provided by \\ emergency services in Recife, Pernambuco, \\ Brazil
}

Antonio da Cruz Gouveia Mendes 1

José Luís Corrêa Amaral de Araújo Júnior 2

Betise Mery Alencar Souza Furtado 3

Petra Oliveira Duarte 4

Renata Florêncio Santiago 5

Tadeu Rodrigues da Costa 6

1-5 Departamento de Saúde Coletiva. Centro de Pesquisas Aggeu Magalhães. Fundação Oswaldo Cruz. Av. Moraes Rego, s. n Cidade Universitária. Recife, PE, Brasil. CEP: 50.670-420. E-mail: amendes@cpqam.fiocruz.br

6 Programa de Pós-Graduação em Biometria e Estatística Aplicada Universidade Federal Rural de Pernambuco. Recife, PE, Brasil.

\section{Resumo}

Objetivos: avaliar a satisfação dos usuários em relação à qualidade do atendimento nas grandes emergências do Recife, Pernambuco, Brasil.

Métodos: trata-se de um estudo descritivo de corte transversal, realizado nas três maiores emergências do município: Hospital da Restauração, Hospital Getúlio Vargas e Hospital Otávio de Freitas. Foram entrevistados 420 usuários (5\% dos atendimentos semanais), através da aplicação de questionários fechados, buscando-se uma relação entre variáveis independentes e o grau de satisfação do usuário.

Resultados: o perfil predominante dos usuários foi de uma população adulta jovem (46,7\%), do sexo feminino $(59,8 \%)$, com baixa escolaridade $(61,6 \%)$, dona de casa ou desempregada (62,3\%) e moradora da região metropolitana ( $83 \%$ ). A insatisfação com as condições oferecidas esteve presente em $55,2 \%$ dos usuários, sendo ainda maior entre aqueles de causa externas da emergência da Restauração (77,1\%). As dimensões da qualidade pior avaliadas foram o conforto oferecido, $56 \%$ de insatisfação, e a priorização na assistência ao usuário através dos tempos de espera, com $48,1 \%$.

Conclusão: os resultados demonstraram uma avaliação crítica dos usuários. Percebe-se uma nítida variação de resultados entre as três unidades. A avaliação positiva em relação ao trabalho dos profissionais traz um alento sobre as possibilidades, porém os demais resultados evidenciam que muito há por fazer para qualificação do atendimento de emergência.

Palavras-chave Avaliação em saúde, Serviços de emergência, Satisfação do usuário 


\section{Introdução}

A avaliação de serviços de saúde é uma área já consolidada em suas várias linhas de abordagens. A utilização de indicadores de avaliação econômica, tecnológica e de qualidade da atenção em saúde (acesso, utilização, cobertura, eficácia, satisfação do usuário, objetivos, estrutura, processo, resultados, entre outros) é hoje reconhecida e amplamente divulgada. 1

Apesar de ser uma preocupação antiga, verificase uma vigorosa retomada a esse tema, considerando a avaliação um passo necessário quando se estabelece a meta de buscar a qualidade na atenção à saúde. ${ }^{2}$ A novidade do movimento contemporâneo é colocar em primeiro plano a opinião do usuário como um aspecto determinante no julgamento da qualidade. 3

O conceito de qualidade desenvolvido por Donabedian ${ }^{4}$ permitiu avançar no sentido de incorporar os não especialistas - no caso, os pacientes na definição de parâmetros e na mensuração da qualidade dos serviços. A satisfação do paciente é de fundamental importância como medida de qualidade da atenção, porque proporciona informação sobre o êxito do provedor em alcançar os valores e expectativas do paciente, que são temas em que esses são as maiores autoridades. 5

O processo de utilização dos serviços de saúde é definido como a resultante da interação de comportamento do indivíduo que procura cuidados e do profissional que o conduz dentro desse sistema. ${ }^{6}$ As características individuais dos prestadores, dos profissionais e dos usuários são apontadas como fatores que induzem a demanda a serviços de saúde, sendo, portanto, produto da interação entre clientes, prestadores de cuidados de saúde e sistema de saúde.7

No Brasil, as pesquisas de satisfação do usuário se tornaram mais frequentes a partir da segunda metade da década de 1990, em função do processo de redemocratização do país e do surgimento de movimentos de luta pelos direitos sociais e, especificamente, do acesso a políticas públicas de saúde, o que pode configurar o surgimento das formulações nessa área de pesquisa. ${ }^{8,9}$ Entre os estudos mais recentes realizados no país, os que se sobressaem pela sua dimensão são "A saúde na opinião dos brasileiros", encomendada pelo Conselho Nacional de Secretários de Saúde em parceria com o Ministério da Saúde, 8 e a pesquisa de Gouveia et al.,10 "Health care user satisfaction in Brazil", ambas realizadas em 2003, nas quais estes autores analisaram a satisfação dos usuários com o sistema de saúde. Porém, poucos são os estudos de satisfação do usuário com a assistência de urgência, ${ }^{11,12}$ o que motiva e justifica o estudo da satisfação dos usuários das urgências.

Considerando o exposto, este estudo parte do pressuposto que o componente da ação humana é determinante de todo processo do cuidado da saúde, seja ela a ação do profissional finalístico, seja a ação do usuário que busca o serviço. Para isto, além de definir as dimensões da qualidade a serem avaliadas, teve-se como preocupação ouvir os usuários, como principais atores sociais envolvidos nesse processo. Portanto, tem como objetivo avaliar a satisfação dos usuários sobre a qualidade do atendimento nas grandes emergências da cidade de Recife, Pernambuco, Brasil, quanto às condições oferecidas, percepção do atendimento dos profissionais e dimensões da qualidade.

\section{Métodos}

Estudo descritivo de corte transversal sobre a qualidade da assistência de urgência através da avaliação da satisfação dos usuários dos três maiores Hospitais Públicos de Urgência e Emergência do Recife, Hospital da Restauração (HR), Hospital Getúlio Vargas (HGV) e Hospital Otávio de Freitas (HOF), todos sob gestão estadual.

Esses hospitais contam com 469 médicos e 97 enfermeiros para o atendimento de urgência (prontoatendimento e emergência), realizando em média 2800 atendimentos semanais, num total de 8400 nos três hospitais.

Considerando as características das emergências dos três hospitais, o volume médio de 8400 atendimentos semanais nas três emergências, o interesse em manter uma amostra aleatória com erro absoluto de até $5 \%$ - o total de usuários entrevistados de 420 ( $5 \%$ dos atendimentos semanais) torna-se estatisticamente representativo do universo de usuários atendidos naquelas emergências. Esse tamanho de amostra permitiu estudar as características de interesse com erro absoluto inferior a $2 \%(1,36 \%)$, o que contempla a realização de análises estatísticas e inferências a partir dos resultados.

Para elaboração das dimensões a serem avaliadas tomou-se como referencial teórico e metodológico o Projeto de Metodologia de Avaliação do Desempenho do Sistema de Saúde Brasileiro (PROADESS), ${ }^{13}$ a Política de Qualificação da Atenção à Saúde no Sistema Único de Saúde (QualiSUS) ${ }^{14}$ e a Política Nacional de Humanização (HumanizaSUS). 15 Esta pesquisa tem como referencial analítico dos resultados essas duas políticas oficiais 
vigentes, que têm como objetivo elevar o nível de qualidade da assistência à saúde prestada à população pelo Sistema Único de Saúde, em particular do sistema de urgência do país.

A partir desses referenciais teóricos e políticos foram definidas as dimensões da qualidade que privilegiam a perspectiva do usuário como cidadão e os serviços de saúde como ação do Estado: a) confidencialidade das informações dadas pelos usuários; b) privacidade no atendimento, referente à não exposição do paciente a outros; c) direito à informação prestada pelos profissionais; d) conforto, relacionado às condições em que o atendimento foi realizado; e) dignidade e cortesia por parte dos profissionais durante a estada do paciente na emergência; f) tempo de espera para realização de exames e para o atendimento; e g) percepção da qualidade da assistência clínica realizada pelos médicos e equipe de enfermagem.

Além dessa avaliação, as mesmas questões contidas nos questionários propiciaram a análise da estrutura e do processo de trabalho nas urgências dos hospitais. Como estrutura foram consideradas as condições oferecidas pelos hospitais através das características do ambiente e da organização hospitalar para o bom atendimento aos usuários e a boa prática profissional. Como processo foi observado pelos usuários o trabalho dos profissionais médicos, enfermeiros e da recepção, ou seja, a ação e interrelação humana na atenção à saúde do usuário.

A coleta dos dados ocorreu no período de outubro de 2006 a janeiro de 2007. Para a análise da satisfação do usuário com o atendimento prestado foi utilizado questionário com perguntas fechadas. $\mathrm{O}$ perfil encontrado permitiu trabalhar a relação entre as variáveis independentes e o grau de satisfação de acordo com as dimensões da qualidade analisadas.

Para verificar a existência de diferenças significativas nas notas com relação às características dos usuários, utilizou-se o teste de Kruskal-Wallis para testes que envolviam mais de duas amostras independentes. Quando esse teste indicou existência de diferenças significativas, utilizou-se o teste de Mann-Whitney para comparar as amostras duas a duas. O nível de significância adotado para esses testes foi de $5 \% .{ }^{16}$

Para definição do que seria satisfação dos usuários foram levados em consideração aqueles usuários que estavam no processo de realização dos cuidados médicos e de enfermagem, cujo "sentimento de gratidão" pode influenciar a percepção dos serviços públicos, dificultando uma visão mais crítica. 1,5,9 Portanto, os critérios de aprovação deveriam ser rigorosos e baseados na literatura especí- fica. Na revisão bibliográfica, apesar da diversidade de métodos e instrumentos, a satisfação é estabelecida quando o usuário avalia como bom ou muito bom, e alta ou muito alta satisfação, $8,12,17$ ou quando o usuário avalia com nota igual ou superior a sete. 11,18 Neste sentido, para arbitrar sobre o que seria a satisfação do usuário com o atendimento de urgência, foi definido que seriam as avaliações com notas iguais ou superiores a sete. As notas foram agregadas em médias aritméticas para cada categoria avaliada, e dispostas segundo frequência de usuários para cada nível ou conceito de avaliação.

O projeto foi aprovado pelo Comitê de Ética do Centro de Pesquisas Aggeu Magalhães (CPqAM), sendo autorizada sua realização pelos gestores estaduais.

\section{Resultados}

Foram entrevistados 70 usuários do pronto-atendimento e 70 da emergência, 140 por hospital, totalizando 420 usuários nos três hospitais, nos turnos diurnos e noturnos, 10 usuários por turno, dos sete dias da semana.

Entre os usuários entrevistados há uma predominância do sexo feminino, $59,8 \%$, e em relação à faixa etária, a maioria encontra-se na faixa de $20 \mathrm{a}$ 39 anos (46,7\%). Quanto à escolaridade, a grande maioria tem ensino fundamental $(48,3 \%)$ ou médio $(27,4 \%)$. O restante se dividiu entre sem escolaridade, $13,3 \%$, com nível superior, 4,5\%, e 6,4\% dos usuários não responderam a essa questão.

Os entrevistados em sua maioria são donas de casa $(31,9 \%)$, seguido dos desempregados, com $30,4 \%$. Os empregados com vínculos formais representaram $24,5 \%$ dos entrevistados e os estudantes $6,4 \%$. Com relação ao local de moradia, $49 \%$ dos usuários são oriundos do Recife e $34 \%$ de outros municípios da Região Metropolitana do Recife.

Quanto ao meio de transporte para chegar até o hospital, observa-se que $32,4 \%$ utilizaram o carro; $33,2 \%$ chegam ao hospital de ônibus ou kombi, a pé ou motocicleta; $32,8 \%$ dos usuários chegam de ambulância, e destes 7,6\% em ambulância do Serviço de Atendimento Móvel de Urgência (SAMU).

Dos usuários atendidos, $38 \%$ chegam com encaminhamento formal e $62 \%$ acessam as emergências por demanda espontânea. A presença de acompanhante é relatada por 79,5\% dos usuários.

A avaliação dos usuários evidencia que a satisfação com o trabalho dos profissionais é bem superior à satisfação com as condições oferecidas ao usuário (Tabela 1). Os hospitais com maiores níveis 
de insatisfação com as condições oferecidas são os da Restauração (60,7\%) e Otávio de Freitas (57,9\%). Quanto ao trabalho dos profissionais, é alta a satisfação no Getúlio Vargas (77,9\%) e Otávio de Freitas (77,1\%). A Restauração tem um percentual significativo de insatisfação $(42,1 \%)$.
Ao correlacionar as condições oferecidas pelo hospital e o trabalho dos profissionais com a faixa etária dos usuários (Tabela 2) percebe-se que os usuários da faixa etária 20 a 39 anos (adultos jovens) apresentam uma maior insatisfação tanto em relação às condições oferecidas quanto em relação à avaliação do trabalho dos profissionais.

Tabela 1

Avaliação das condições oferecidas ao usuário e trabalho dos profissionais, segundo as emergências dos Hospitais da Restauração, Otávio de Freitas e Getúlio Vargas. Recife, Pernambuco, 2007.

\begin{tabular}{|c|c|c|c|c|c|c|c|c|c|}
\hline \multirow{2}{*}{ Dimensões } & \multirow{2}{*}{ Hospitais } & \multicolumn{2}{|c|}{ Insatisfeito } & \multicolumn{2}{|c|}{ Satisfeito } & \multirow{2}{*}{ Total } & \multirow{2}{*}{$\begin{array}{l}\text { Nota } \\
\text { Média }\end{array}$} & \multirow{2}{*}{ Hipótese } & \multirow{2}{*}{$p$} \\
\hline & & $\mathrm{n}$ & $\%$ & $\mathrm{n}$ & $\%$ & & & & \\
\hline Condições & $H R^{*}$ & 85 & 60,7 & 55 & 39,3 & 140 & 6,4 & $\mathrm{a}=\mathrm{b}=\mathrm{c} * * * *$ & 0,034 \\
\hline \multirow[t]{4}{*}{ oferecidas } & HOF** & 81 & 57,9 & 59 & 42,1 & 140 & 6,5 & $\mathrm{a}=\mathrm{b} * * * * *$ & 0,502 \\
\hline & $\mathrm{HGV} * * *$ & 66 & 47,1 & 74 & 52,9 & 140 & 6,9 & $a=c * * * * *$ & 0,010 \\
\hline & Total & 232 & 55,2 & 188 & 44,8 & 420 & & $\mathrm{~b}=\mathrm{c} * * * * *$ & 0,083 \\
\hline & $H R^{*}$ & 59 & 42,1 & 81 & 57,9 & 140 & 7,4 & $\mathrm{a}=\mathrm{b}=\mathrm{c} * * * *$ & 0,024 \\
\hline Trabalho dos & $\mathrm{HOF}^{* *}$ & 32 & 22,9 & 108 & 77,1 & 140 & 7,8 & $\mathrm{a}=\mathrm{b} * \star * \star *$ & 0,032 \\
\hline \multirow[t]{2}{*}{ profissionais } & $\mathrm{HGV} * * *$ & 31 & 22,1 & 109 & 77,9 & 140 & 7,9 & $\mathrm{a}=\mathrm{c} * * * * *$ & 0,013 \\
\hline & Total & 122 & 29,1 & 298 & 71,0 & 420 & & $\mathrm{~b}=\mathrm{c} * * * * *$ & 0,520 \\
\hline
\end{tabular}

* Hospital da Restauração; ** Hospital Otávio de Freitas; *** Hospital Getúlio Vargas; **** Teste de Kruskal-Wallis; ***** Teste de Mann-Whitney.

Tabela 2

Avaliação das condições oferecidas ao usuário e trabalho dos profissionais segundo a faixa etária dos usuários nas emergências dos Hospitais da Restauração, Getúlio Vargas e Otávio de Freitas. Recife, Pernambuco, 2007.

\begin{tabular}{|c|c|c|c|c|c|c|c|c|c|}
\hline \multirow{2}{*}{ Dimensões } & \multirow{2}{*}{ Faixa Etária } & \multicolumn{2}{|c|}{ Insatisfeito } & \multicolumn{2}{|c|}{ Satisfeito } & \multirow{2}{*}{ Total } & \multirow{2}{*}{$\begin{array}{l}\text { Nota } \\
\text { Média }\end{array}$} & \multirow{2}{*}{ Hipótese } & \multirow{2}{*}{$p$} \\
\hline & & $\mathrm{n}$ & $\%$ & $\mathrm{n}$ & $\%$ & & & & \\
\hline \multirow{10}{*}{$\begin{array}{l}\text { Condições } \\
\text { oferecidas }\end{array}$} & 13 a 19 anosa & 8 & 47,1 & 9 & 52,9 & 17 & 7,0 & $\mathrm{a}=\mathrm{b}=\mathrm{c}=\mathrm{d}^{*}$ & 0,023 \\
\hline & & & & & & & & $a=b * *$ & 0,091 \\
\hline & 20 a 39 anosb & 115 & 62,5 & 69 & 37,5 & 184 & 6,2 & $a=c * *$ & 0,509 \\
\hline & & & & & & & & $a=d * *$ & 0,621 \\
\hline & 40 a 59 anosc & 68 & 55,3 & 55 & 44,7 & 123 & 6,7 & $\mathrm{~b}=\mathrm{c} * *$ & 0,016 \\
\hline & & & & & & & & $\mathrm{b}=\mathrm{d} * *$ & 0,026 \\
\hline & 60 anos e maisd & 32 & 51,6 & 30 & 48,4 & 62 & 6,8 & $c=d * *$ & 0,742 \\
\hline & Total & 223 & 57,8 & 163 & 42,2 & 386 & & & \\
\hline & 13 a 19 anosa & 2 & 11,8 & 15 & 88,2 & 17 & 8,2 & $\mathrm{a}=\mathrm{b}=\mathrm{c}=\mathrm{d}^{*}$ & 0,038 \\
\hline & & & & & & & & $a=b * *$ & 0,094 \\
\hline \multirow{6}{*}{$\begin{array}{l}\text { Trabalho dos } \\
\text { profissionais }\end{array}$} & 20 a 39 anosb & 65 & 35,3 & 119 & 64,7 & 184 & 7,4 & $a=c * *$ & 0,310 \\
\hline & & & & & & & & $a=d * *$ & 0,821 \\
\hline & 40 a 59 anosc & 34 & 27,6 & 89 & 72,4 & 123 & 7,8 & $\mathrm{~b}=\mathrm{c} * *$ & 0,080 \\
\hline & & & & & & & & $\mathrm{b}=\mathrm{d} * *$ & 0,017 \\
\hline & 60 anos e maisd & 152 & 4,2 & 47 & 75,8 & 62 & 8,0 & $c=d * *$ & 0,292 \\
\hline & Total & 116 & 30,1 & 270 & 70,0 & 386 & & & \\
\hline
\end{tabular}

* Teste de Kruskal-Wallis; ** Teste de Mann-Whitney. 
Ao avaliar as condições oferecidas de acordo com a queixa do usuário (Tabela 3 ) percebe-se que os usuários do HR com queixas de causas externas apresentam um nível de insatisfação extremamente elevado (72,1\%), bem superior aos demais hospitais. Ao contrário do HR, os usuários do HOF e HGV com maior insatisfação foram os com outras causas como motivo de procura às emergências.
Em relação ao trabalho dos profissionais (Tabela 4), os usuários que procuraram o HR com queixas de causas externas têm um nível de insatisfação $(54,1 \%)$ bem superior aos do HOF e HGV, 16,1\% e $14,3 \%$ respectivamente. Os usuários do HOF e HGV apresentam uma alta satisfação tanto nos usuários com causas externas como nos com outras causas, sendo essa variável discriminadora somente em relação ao HR.

Tabela 3

Avaliação da satisfação dos usuários com as condições oferecidas pelo hospital, segundo tipo de queixa dos usuários e emergências dos Hospitais da Restauração, Otávio de Freitas e Getúlio Vargas. Recife, Pernambuco, 2007.

\begin{tabular}{|c|c|c|c|c|c|c|c|c|c|}
\hline \multirow{2}{*}{ Tipo de Queixa } & \multirow{2}{*}{ Hospital } & \multicolumn{2}{|c|}{ Insatisfeito } & \multicolumn{2}{|c|}{ Satisfeito } & \multirow{2}{*}{ Total } & \multirow{2}{*}{$\begin{array}{l}\text { Nota } \\
\text { Média }\end{array}$} & \multirow{2}{*}{ Hipótese } & \multirow{2}{*}{$p$} \\
\hline & & $\mathrm{n}$ & $\%$ & $\mathrm{n}$ & $\%$ & & & & \\
\hline Condições & $\mathrm{HR}^{*}$ & 44 & 72,1 & 17 & 27,9 & 61 & 6,0 & $\mathrm{a}=\mathrm{b}=\mathrm{c} * * * *$ & 0,002 \\
\hline \multirow[t]{4}{*}{ oferecidas } & HOF** & 15 & 48,4 & 16 & 51,6 & 31 & 6,7 & $\mathrm{a}=\mathrm{b} * * * * *$ & 0,043 \\
\hline & $\mathrm{HGV} * * *$ & 10 & 35,7 & 18 & 64,3 & 28 & 7,3 & $\mathrm{a}=\mathrm{c} * * * * *$ & 0,001 \\
\hline & Total & 69 & 57,5 & 51 & 42,5 & 120 & & $\mathrm{~b}=\mathrm{c} * * * * *$ & 0,354 \\
\hline & $H R^{*}$ & 41 & 51,9 & 38 & 48,1 & 79 & 6,7 & $\mathrm{a}=\mathrm{b}=\mathrm{c} * * * *$ & 0,294 \\
\hline Outras & HOF** & 66 & 60,6 & 43 & 39,5 & 109 & 6,4 & & \\
\hline \multirow[t]{2}{*}{ causas } & $\mathrm{HGV} * * *$ & 56 & 50,0 & 56 & 50,0 & 112 & 6,8 & & \\
\hline & Total & 163 & 54,3 & 137 & 45,7 & 300 & & & \\
\hline
\end{tabular}

*Hospital da Restauração; ** Hospital Otávio de Freitas; *** Hospital Getúlio Vargas; **** Teste de KruskalWallis; *****Teste de Mann- Whitney.

Tabela 4

Avaliação da satisfação dos usuários com o trabalho dos profissionais, segundo tipo de queixa dos usuários e emergências dos Hospitais da Restauração, Otávio de Freitas e Getúlio Vargas. Recife, Pernambuco, 2007.

\begin{tabular}{|c|c|c|c|c|c|c|c|c|c|}
\hline \multirow{2}{*}{ Tipo de Queixa } & \multirow{2}{*}{ Hospital } & \multicolumn{2}{|c|}{ Insatisfeito } & \multicolumn{2}{|c|}{ Satisfeito } & \multirow{2}{*}{ Total } & \multirow{2}{*}{$\begin{array}{l}\text { Nota } \\
\text { Média }\end{array}$} & \multirow{2}{*}{ Hipótese } & \multirow{2}{*}{$p$} \\
\hline & & $\mathrm{n}$ & $\%$ & $\mathrm{n}$ & $\%$ & & & & \\
\hline Causas & $\mathrm{HR}^{*}$ & 33 & 54,1 & 28 & 45,9 & 61 & 6,7 & $\mathrm{a}=\mathrm{b}=\mathrm{c} * * * *$ & $<0,001$ \\
\hline \multirow[t]{4}{*}{ externas } & HOF** & 5 & 16,1 & 26 & 83,9 & 31 & 8,1 & $a=b * * * * *$ & $<0,001$ \\
\hline & $\mathrm{HGV}^{*} * *$ & 4 & 14,3 & 24 & 85,7 & 28 & 8,1 & $\mathrm{a}=\mathrm{c} * * * * *$ & 0,002 \\
\hline & Total & 42 & 35,0 & 78 & 65,0 & 120 & & $\mathrm{~b}=\mathrm{c} * * * * *$ & 0,891 \\
\hline & $\mathrm{HR}^{*}$ & 26 & 32,9 & 53 & 67,1 & 79 & 7,9 & $\mathrm{a}=\mathrm{b}=\mathrm{c} * * * *$ & 0,666 \\
\hline Outras & $\mathrm{HOF}^{* *}$ & 27 & 24,8 & 82 & 75,2 & 109 & 7,8 & & \\
\hline \multirow[t]{2}{*}{ causas } & $\mathrm{HGV} * * *$ & 27 & 24,1 & 85 & 75,9 & 112 & 7,9 & & \\
\hline & Total & 80 & 26,7 & 220 & 73,3 & 300 & & & \\
\hline
\end{tabular}

* Hospital da Restauração; ** Hospital Otávio de Freitas; *** Hospital Getúlio Vargas; **** Teste de KruskalWallis; ***** Teste de Mann- Whitney. 
Os resultados das dimensões da qualidade nos três hospitais (Tabela 5) apresentam uma grande satisfação dos usuários com a confidencialidade das informações prestadas e uma alta insatisfação com o conforto e os tempos de espera.

Tabela 5

Avaliação da satisfação dos usuários segundo as dimensões da qualidade e emergências dos Hospitais da Restauração, Otávio de Freitas e Getúlio Vargas. Recife-Pernambuco, 2007.

\begin{tabular}{|c|c|c|c|c|c|c|c|c|c|c|}
\hline \multirow{3}{*}{$\begin{array}{l}\text { Dimensões da } \\
\text { Qualidade }\end{array}$} & \multicolumn{6}{|c|}{ Satisfação do Usuário } & & & \multirow{3}{*}{ Hipótese } & \multirow{3}{*}{$p$} \\
\hline & \multicolumn{2}{|c|}{$\begin{array}{l}\text { Hospital da } \\
\text { Restauração }\end{array}$} & \multicolumn{2}{|c|}{$\begin{array}{l}\text { Hospital Otávio } \\
\text { de Freitas }\end{array}$} & \multicolumn{2}{|c|}{$\begin{array}{l}\text { Hospital Getúlio } \\
\text { Vargas }\end{array}$} & \multicolumn{2}{|c|}{ Total } & & \\
\hline & $\mathrm{n}$ & $\%$ & $\mathrm{n}$ & $\%$ & $n$ & $\%$ & $\mathrm{n}$ & $\%$ & & \\
\hline $\begin{array}{l}\text { Confidencialidade } \\
\text { das informações }\end{array}$ & 111 & 79,3 & 122 & 87,1 & 118 & 84,3 & 351 & 83,6 & $a=b=c^{*}$ & 0,052 \\
\hline $\begin{array}{l}\text { Privacidade no } \\
\text { atendimento }\end{array}$ & 75 & 54,4 & 80 & 58,0 & 94 & 67,6 & 249 & 60,0 & $a=b=c^{*}$ & 0,223 \\
\hline $\begin{array}{l}\text { Direito à } \\
\text { informação }\end{array}$ & 80 & 57,1 & 90 & 64,3 & 92 & 65,7 & 262 & 62,4 & $\begin{array}{l}a=b=c^{*} \\
a=b=c^{*}\end{array}$ & $\begin{array}{r}0,171 \\
<0,001\end{array}$ \\
\hline Conforto & 55 & 39,3 & 51 & 36,4 & 79 & 56,4 & 185 & 44,1 & $\begin{array}{l}a=c^{* *} \\
b=c^{* *}\end{array}$ & $\begin{array}{l}<0,001 \\
<0,001\end{array}$ \\
\hline $\begin{array}{l}\text { Dignidade e } \\
\text { cortesia }\end{array}$ & 80 & 57,1 & 91 & 65,0 & 91 & 65,0 & 262 & 62,4 & $a=b=c^{*}$ & 0,303 \\
\hline $\begin{array}{l}\text { Tempos de } \\
\text { espera }\end{array}$ & 63 & 45,0 & 79 & 56,4 & 76 & 54,3 & 218 & 51,9 & $a=b=c^{*}$ & 0,164 \\
\hline $\begin{array}{l}\text { Assistência } \\
\text { clínica aos usuários }\end{array}$ & 76 & 54,3 & 94 & 67,1 & 93 & 66,4 & 263 & 62,6 & $a=b=c^{*}$ & 0,100 \\
\hline
\end{tabular}

*Teste de Kruskal-Wallis; ** Teste de Mann- Whitney.

Em todas as dimensões avaliadas, com exceção da confidencialidade, o HR apresenta uma baixa satisfação dos usuários. O HGV é o melhor avaliado, embora tenha níveis baixos de satisfação para conforto e os tempos de espera semelhantes ao HOF, este último, porém, tem também uma baixa satisfação para a privacidade no atendimento.

Examinando-se os resultados em cada uma das dimensões e as questões que a compõem, foi observado que: a) a confidencialidade das informações tem na confiança dos usuários em contar seus problemas aos médicos a questão melhor avaliada dessa dimensão, satisfação de $90,5 \%$. A privacidade do atendimento tem uma insatisfação de 45,7 e 42,05 no HR e HOF; b) o direito à informação, a dignidade e cortesia no atendimento e a qualidade da assistência clínica aos usuários tem avaliações semelhantes, com grau de satisfação em torno de $65 \%$ no HOF e HGV e menor satisfação dos usuários do HR (em torno de $55 \%$ ).

As questões melhor avaliadas em relação ao direito à informação foram as informações repassadas pelos médicos e a facilidade de achar os vários setores dos hospitais, e a pior avaliada foi a disponibilidade dos funcionários para dar orientações, com 32,9\% de insatisfação.

Quanto à dignidade e cortesia no atendimento, a questão com maior satisfação foi a possibilidade da presença do acompanhante (80\%); outras com satisfação superior a $70 \%$ foram a facilidade de entrar no hospital, a gentileza dos profissionais e a disponibilidade dos profissionais para o atendimento. A maior insatisfação (55,3\%) dos usuários foi a possibilidade de fazer reclamações e ser ouvido dentro do hospital.

As questões referentes à percepção da qualidade da assistência clínica com nível de satisfação superior a $80 \%$ foram a paciência do médico para escutar os problemas de saúde no detalhamento do exame clínico e a realização dos cuidados da enfermagem. As maiores insatisfações foram com a ausência da chefia de enfermagem e a quantidade 
de médicos, ambas com $48 \%$ de insatisfação. A quantidade de profissionais de enfermagem e os cuidados de enfermagem na higienização apresentaram uma insatisfação superior a $35 \%$.

O conforto não é bem avaliado em nenhum dos três hospitais, tendo o HOF e o HR os mais altos níveis de insatisfação, 63,6\% e 60,7\% respectivamente. As questões que mais influenciam na insatisfação com o conforto são a quantidade e conforto das cadeiras, a conservação e a limpeza do mobiliário e macas. As questões com satisfação superior a $70 \%$ foram a qualidade das refeições e a limpeza dos hospitais.

Em relação aos tempos de espera para ser atendido e para realização de exames, os hospitais apresentaram uma alta insatisfação, em particular no HR $(55,0 \%)$.

Por último, ao final do questionário foi solicitado que o usuário desse uma nota geral em relação ao atendimento recebido no hospital. Essa nota permitiria realizar a comparação desta avaliação com a avaliação das dimensões estudadas. Os resultados alcançados foram homogêneos entre os hospitais, apresentando uma satisfação de $77 \%$ com o atendimento das emergências.

\section{Discussão}

O perfil predominante dos usuários das três grandes emergências do Recife é de uma população adulta jovem, do sexo feminino, com baixa escolaridade, dona de casa ou desempregada, moradora da região metropolitana. A maior presença de mulheres, como usuárias e como acompanhantes, nos serviços de saúde, 59,8\% neste estudo, demonstra a mulher como a maior usuária das emergências, como em outros estudos, 19-21 e sua importância no papel de cuidadora da saúde da família.

Outra característica da clientela dessas emergências é que os pacientes chegam às unidades por demanda espontânea e em meios de locomoção inapropriados para os casos de emergência, evidenciando que estes hospitais atendem um grande número de pacientes com queixas de baixa complexidade, superlotando as emergências com casos que poderiam ser resolvidos em Serviços de Pronto Atendimento, problema que ultrapassa os limites da gestão hospitalar. Como nesses hospitais estão as três maiores emergências gerais do Estado de Pernambuco, esse tipo de assistência nos referidos hospitais é um desperdício de recursos humanos e tecnológicos de alta complexidade e um desvirtuamento de sua missão institucional.22

Assim como encontrado por O'Dwyer et al.,23 na avaliação da qualidade do atendimento ficou evidenciado que o trabalho dos profissionais é o componente melhor avaliado pelos usuários. Nascimento 24 também referiu a insatisfação com as condições oferecidas. Os hospitais avaliados, apesar de contarem com uma incorporação tecnológica compatível com seu grau de complexidade, apresentam precárias condições de atendimento aos usuários, com uma estrutura arquitetônica ultrapassada e mobiliário não condizente com a situação vivida pelo usuário.

Os adultos jovens apresentam uma maior insatisfação com as condições oferecidas e com o trabalho dos profissionais e essa faixa etária representa $48 \%$ dos usuários. A criticidade desses usuários determina um maior nível de insatisfação na avaliação. Outro estudo realizado para identificar fatores associados com a satisfação de pacientes internados 25 evidenciou que um dos mais fortes preditores de satisfação era a idade mais avançada do paciente.

A altíssima insatisfação com as condições oferecidas e o trabalho dos profissionais dos usuários com queixas de causas externas do HR não é acompanhada da mesma avaliação dos usuários com o mesmo tipo de queixa no HOF e HGV; ao contrário, nesses hospitais a maior insatisfação está entre os usuários com outras causas de procura ao hospital. Como o número de usuários com causas externa do HR é mais que o dobro em relação aos outros hospitais e como o HR apresenta um perfil de maior complexidade, talvez esteja na "gravidade" das causas externas, que leva a uma menor autonomia dos usuários desse hospital, a "justificativa" para uma maior insatisfação. Contudo, os resultados não coincidem com o achado em recente estudo em hospital de emergência de Fortaleza, que indica elevado nível de satisfação apesar da demora do atendimento. 12

$\mathrm{Na}$ análise das dimensões da qualidade ficou evidenciado que apenas a confidencialidade das informações ultrapassa um nível de satisfação superior a $70 \%$. Apesar da grande insatisfação com o tempo de espera, tal insatisfação foi superada pela avaliação do conforto, assim como chama atenção a alta insatisfação com a privacidade no atendimento. Em outro estudo, o tempo de espera também foi motivo de grande insatisfação. ${ }^{12}$ Como este estudo refere-se a unidades de emergência e tem uma boa satisfação com a facilidade em entrar no hospital, a pouca satisfação com os tempos de espera refere-se mais aos tempos internos para realização dos vários procedimentos.

A avaliação dos usuários em relação ao conforto e tempos de espera corrobora a evidência de que nesses serviços pacientes estão "internados" por 
longo período em macas nos corredores das emergências, demonstrando que políticas de qualificação não estão sendo plenamente implantadas e que a gestão da assistência clínica não está tendo a efetividade necessária. 22,26

As questões componentes do questionário que mais contribuem para uma maior satisfação dos usuários são referentes à ação dos profissionais médicos, da equipe de enfermagem e da recepção, principalmente as referentes aos cuidados dos profissionais com os pacientes. Mas, também chama atenção a avaliação positiva de questões como a facilidade em entrar no hospital, a limpeza do ambiente, a refeição servida e a possibilidade da presença do acompanhante na emergência. A presença do acompanhante também foi relatada em outro estudo sobre satisfação das mulheres com a assistência ao parto, 17 como fator que contribui para a satisfação das parturientes. A satisfação com tais questões evidencia a evolução na qualificação, provavelmente devido ao início da implantação de políticas como o QualiSUS14 e HumanizaSUS15 nesses hospitais.

A quantidade e conforto das cadeiras, a conservação e limpeza do mobiliário e macas, o barulho no ambiente hospitalar, os tempos de espera, a pouca possibilidade de fazer reclamação e ser ouvido, a presença do chefe de enfermagem na orientação de condutas e a quantidade de médicos na emergência são questões que contribuem para a maior insatisfação do usuário. A queixa em relação à quantidade de médicos não tem uma explicação tão evidente, porque os hospitais observados no estudo contam com 20 a 30 médicos, além de residentes, de plantão em cada turno.

A avaliação dos usuários no momento do atendimento de urgência exige uma grande valorização da sua análise crítica e os resultados demonstram que esses hospitais têm um longo caminho a percorrer para sua qualificação. Os resultados demonstram que apesar da grande relevância social do trabalho e dos resultados clínicos alcançados por esses hospitais, a avaliação fica comprometida por questões aparentemente simples, mas que envolvem principalmente um grande investimento gerencial.

A qualificação da atenção às urgências preconizadas pelo QualiSUS e HumanizaSUS do Ministério da Saúde, 14,15 referencial analítico desta pesquisa, pressupõe mudanças internas com a implantação do acolhimento, humanização, reorganização do fluxo e dos espaços internos de acordo com a gravidade do caso e a priorização dos leitos do hospital para o usuário da emergência. Essa qualificação exige uma mudança de atitude gerencial e tem como elemento central a ação humana através da participação dos trabalhadores desses hospitais na sua implantação. Como o trabalho dos profissionais obteve a melhor avaliação constatada nessa pesquisa, apesar da possibilidade do "viés de gratidão", percebe-se a existência de alguma condição interna necessária para essas mudanças nos hospitais estudados.

As unidades de urgência são uma importante porta de entrada no sistema de saúde e a qualificação da assistência de urgência vai além do ambiente hospitalar. A melhoria da qualidade pressupõe a regulação do Sistema de Urgência. Regulando a entrada nas emergências e redistribuindo os casos de menor complexidade para a rede de assistência às urgências organizada por nível de complexidade, bem como realizando uma efetiva regulação dos leitos hospitalares, se possibilitaria o atendimento e a internação dos que chegam às emergências de acordo com a especialização, grau de complexidade e distribuição espacial da rede hospitalar. Com isto, diminuiria o tempo de permanência dos pacientes e seriam desobstruídas as emergências de alta complexidade, permitindo a qualificação interna das unidades de emergência.

A avaliação muito positiva, quando solicitada uma nota geral ao atendimento nos hospitais, resultado muito diferente das demais avaliações realizadas nesse trabalho, demonstra que avaliações gerais, pouco explicitadoras do que se quer avaliar, contribuem muito pouco para se ter uma avaliação mais fidedigna do usuário. Os resultados obtidos nesse trabalho evidenciam que a metodologia adotada foi capaz de captar a (in)satisfação do usuário, por ser discriminadora dos vários aspectos da assistência que se quis avaliar.

Os resultados dessa pesquisa apresentam um nível de satisfação diferente de outros estudos sobre satisfação com o atendimento à urgência, como o realizado no Gea et al., ${ }^{11}$ no Servicio de Urgencia del Centro Médico-Quirúrgico del Hospital Virgen de las Neves, na cidade de Granada, Espanha, em que a proporção de usuários satisfeitos foi de $90 \%$. A investigação de Braga Júnior et al.12 sobre a epidemioloia e o grau satisfação do paciente vítima de trauma músculo-esquelético atendido em hospital de emergência da rede pública brasileira, Fortaleza, Ceará, encontrou uma satisfação de $89 \%$. Outros estudos sobre satisfação com a hospitalização têm avaliações de satisfação superiores a 70\% no Brasil10,17 e superiores a $80 \%$ na América do Sul.20,27 Para entender as diferenças de avaliações encontradas, é preciso considerar a diversidade de métodos, técnicas, instrumentos e dimensões da 
qualidade selecionadas, utilizados nas diversas pesquisas, o que torna as comparações difíceis de serem realizadas. Pesquisar a satisfação no momento

\section{Referências}

1. Deslandes SF. Concepções em pesquisa social: articulações com o campo da avaliação em serviços de saúde. Cad Saúde Pública. 1997; 13: 103-7.

2. Franco SC, Campos GWS. Avaliação da qualidade de atendimento ambulatorial em pediatria em um hospital universitário. Cad Saúde Pública. 1998; 14: 61-70.

3. Serapioni M. Avaliação da qualidade em saúde: a contribuição da sociologia da saúde para a superação da polarização entre a visão dos usuários e a perspectiva dos profissionais de saúde. Saúde Debate. 1999; 23: 81-92.

4. Donabedian A. La calidad de la atención médica. México: La Prensa Médica Mexicana; 1984.

5. Vaistman J, Andrade GRB. Satisfação e responsividade: formas de medir a qualidade e a humanização da assistência à saúde. Ciênc Saúde Coletiva. 2005; 10: 599-613.

6. Travassos C, Martins M. Uma revisão sobre os conceitos de acesso e utilização de serviços de saúde. Cad Saúde Pública. 2004; 20 (Supl. 2): S190-S8.

7. Dutton D. Financial organizational and professional factors affecting health care utilization. Soc Sci Med. 1986; 23: 721-35.

8. Brasil. Conselho Nacional de Secretários de Saúde (CONASS). A saúde na opinião dos brasileiros. Brasília, DF; 2003.

9. Esperidião MA, Trad LAB. Avaliação de satisfação de usuários: considerações teórico-conceituais. Cad Saúde Pública. 2006; 22: 1267-76.

10. Gouveia GC, Souza WV, Luna CF, Souza-Júnior PRB, Szwacwald CL. Health care user satisfaction in Brazil, 2003. Cad Saúde Pública. 2005; 21 (Supl): S109-S18.

11. Gea MT, García MH, Martín JMJ, Cabrera A. Opinión de los usuarios sobre la calidad del Servicio de Urgencias del Centro Médico-Quirúrgico del Hospital Virgen de las Nieves. Rev Calid Asist. 2001; 16: 37-44.

12. Braga Júnior MB, Chagas Neto FA, Porto M A, Barroso TA, Lima ACM, Silva SM, Lopes MWB. Epidemiologia e grau de satisfação do paciente vítima de trauma músculoesquelético atendido em Hospital de Emergência da rede pública brasileira. Acta Ortop Bras. 2005; 13: 137-40.

13. FIOCRUZ (Fundação Oswaldo Cruz). ProjetoDesenvolvimento de metodologia de Avaliação do Desempenho do Sistema de Saúde Brasileiro (PROADESS): relatório final. Rio de Janeiro; 2003.

14. Brasil. Ministério da Saúde. QualiSUS: política de qualificação da atenção à saúde no Sistema Único de Saúde. Brasília, DF; 2003.

15. Brasil. Ministério da Saúde. HumanizaSUS: política nacional de humanização. A humanização como eixo norteador das práticas de atenção e gestão em todas as instâncias do SUS. Brasília, DF; 2004. (Série B. Textos Básicos de Saúde).

Recebido em 25 de janeiro de 2008

Versão final apresentada em 06 de janeiro de 2009

Aprovado em 3 de fevereiro de 2009 da assistência dentro das emergências talvez tenha sido o fator determinante da criticidade dos usuários dessa pesquisa.

16. Vieira S. Bioestatística: tópicos avançados. Rio de Janeiro: Elsevier; 2004.

17. Domingues RMSM, Santos EM, Leal MC. Aspectos da satisfação das mulheres com a assistência ao parto: contribuição para o debate. Cad Saúde Pública. 2004; 20 (Supl 1): S52-S62.

18. Lemme AC, Noronha G, Resende JB. A satisfação do usuário em hospital universitário. Rev Saúde Pública. 1991; 25: 41-6.

19. Galaz C, Godoy R, Cisternas J, Yentzen G, De La Fuente M. Patrones de conducta de los usuários de um Servicio de Urgência adosado a Hospital Tipo 4. Rev Chil Salud Pública. 2004; 8: 84-92.

20. Fernandez MBT, Sancchez MPB, Linares XG. Nível de satisfacción general y analisis del relación médico paciente de los medicos en entrenamiento en las salas de hopitalizacíon de medicina interna. Rev Med Hered. 2003; 14: 17580 .

21. Capilheira MF, Santos IS. Fatores individuais associados à utilização de consultas médicas por adultos. Rev Saúde Pública. 2006; 40: 436-43.

22. Furtado BMA, Araújo Jr JLC, Cavalcanti P. O perfil da emergência do Hospital da Restauração: uma análise dos possíveis impactos após a municipalização dos serviços de saúde. Rev Bras Epidemiol. 2004; 7: 279-89.

23. O’Dwyer G, Matta IEA, Pepe VLE. Avaliação dos serviços hospitalares de emergência do Estado do Rio de Janeiro. Ciênc Saúde Coletiva. 2008; 13: 1637-48.

24. Nascimento VS. Os desafios do trabalhador de enfermagem no setor de emergência de um hospital público. [dissertação]. Rio de Janeiro: Escola de Enfermagem da Universidade do Estado do Rio de Janeiro; 2004.

25. Thi PLN, Briançon S, Empereur F, Guillemin F. Factors determining in pacient satisfaction with care. Soc Sci Med. 2002; 54: 493-504.

26. Gusmão-Filho FAR. Análise de implantação da Política de Qualificação da Atenção à Saúde do Sistema Único de Saúde - Política QualiSUS - em três hospitais do município do Recife. [tese]. Recife: Centro de Pesquisas Aggeu Magalhães da Fundação Oswaldo Cruz; 2008.

27. Alvarado R, Vera A. Evaluación de la satisfacción de usuários de hospitales del sistema nacional de servicios de salud em Chile. Rev Chil Salud Pública. 2001, 5: 81-9. 